and on data which he has been able to garner from acquaintances. He does not attempt to explain or find psychological or political motivation behind the drive to render Soviet mathematics judenfrei. $\mathrm{He}$ is content simply to state the facts as he sees them, and to cite, in an impassioned $J$ 'accuse addressed to the President of the Soviet Academy of Sciences, the names of half-a-dozen leading Soviet mathematicians, the Expert Committee for Mathematics and Mechanics of the Higher Attestation Committee (the body responsible for higher degrees) and the Steklov Institute of Mathematics of the Soviet Academy of Sciences. These few put prestigious persons and bodies, he says, have made Soviet mathematics "a cesspool of scientific unprincipledness".

Granted the conditions described in Freiman's essay, and the resolute official denials that anything in the nature of antiSemitism can exist in the Soviet Union, it would seem virtually impossible for any impartial third party to assess the validity of Freiman's claims. A certain weight should undoubtedly be given to the fact that at the time of writing the essay Freiman himself was neither a refusnik nor an émigré. (Since then, he has filed an application to emigrate to Israel.) Weight, too, should be given to the style of the essay, remarkably free from the introspective self-pity that mars so many samizdat biographies of repression. One piece of evidence mentioned by Freiman is, however, open to checking by the Western reader - the virtual disappearance over the past few years of Jewish surnames from the contributors to the Matematicheskii Sbornik and other leading Soviet mathematical journals. Thanks to the various cover-to-cover translation bureaux, most of these journals are available, in their entirety, in English translations. Those readers who find Freiman's revelations difficult to accept can count for themselves.

Vera Rich is a journalist who specializes in East European science affairs.

\section{Publicity for exotic atoms}

\section{C.J. Batty}

Exotic Atoms '79: Fundamental Interactions and Structure of Matter. Edited by Kenneth Crowe, Jean Duclos, Giovanni Fiorentini and Gabriele Torelli. Pp.403. (Plenum: New York and London, 1980.) $\$ 45, £ 28.35$.

AN EXOTIC atom is an atom in which one of the electrons has been replaced by a negatively charged heavy particle such as a muon $(\mu), \pi$ or K-meson, antiproton $(\overline{\mathrm{p}})$ or by a $\Sigma$-hyperon. It can be formed by stopping a beam of the appropriate particles in a suitable nuclear target. Once the heavy particle has been slowed down to an energy of order $1 \mathrm{keV}$ it can be captured into a Bohr orbit about the nucleus of high principal quantum number $n$. It will then cascade down through its own sequence of atomic levels, first by Auger transitions with the emission of an electron and later by the emission of X-rays. Following each of these transitions it comes closer to the nucleus until at some stage nuclear capture takes place.

Studies of exotic atoms cover a whole range of disciplines from physical chemistry through atomic and solid-state physics to nuclear and elementary particle physics. This diversity of interests and applications is reflected in the 22 articles, of varying length, which appear in Exotic Atoms '79.

The exact mechanism by which the heavy particle is captured to form the exotic atom is not fully understood but the present volume contains an interesting review of this topic by Schneuwly under the heading of "Mesic Chemistry". Four other shorter articles deal with more specific aspects of capture by hydrogen and the properties of muonic-hydrogen atoms. A particularly fascinating review by J. Rafelski from CERN deals with the properties of mesomolecules of hydrogen in which the electron in the hydrogen molecule $\mathrm{H}_{2}{ }^{+}$has been replaced by a muon. In the case of the heavier hydrogen isotopes spontaneous fusion can occur, a process first observed for the system $\mu$ pd at Berkeley in 1956, and there is now considerable interest in the properties of dd and dt mesomolecules.

Rather special examples of exotic atoms are muonium, the system $\mu^{+} \mathrm{e}^{-}$, and positronium, the system $\mathrm{e}^{-} \mathrm{e}^{+}$. The properties of muonium and positronium are reviewed by $V$. Hughes of Yale University, who has worked for many years in this field, and here one is particularly impressed by the elegance of the experiments and the precision of the results. Extensions of the exotic atom picture to consider bound states of quarks to form hadrons are discussed in three include muon capture rates and parity nonconservation in atoms and molecules.

The whole field of muon spin rotation $(\mu \mathrm{SR})$, so named because of its close analogy with nuclear magnetic resonance theoretical articles. Other topics reviewed

(NMR) and electron spin resonance (ESR), has expanded dramatically over the last few years and is now a valuable tool in the study of condensed matter as well as being a topic of study in its own right. It is therefore not surprising that almost half of the book is devoted to this subject. Topics covered include muon diffusion and trapping in solids, aspects of positive muons as impurities in metals, studies of $\mu \mathrm{SR}$ in semiconductors and in ferromagnetic metals, and some features of muonium chemistry.

The book is certainly a valuable contribution to publicizing the existence of a fascinating area of cross-disciplinary research, and will provide an excellent introduction to those meeting the subject for the first time and a valuable reference work for the more expert.

C.J. Batty is on the staff of the Rutherford and Appleton Laboratories at Chilton, Oxfordshire, UK.

\section{Neurophysiological methods in entomology}

\section{Melody Siegler}

Insect Neurophysiological Techniques. By T.A. Miller. Pp.308. (Springer: Heidelberg and New York, 1979.) DM54, \$29.70.

IN Insect Neurophysiological Techniques, T.A. Miller has compiled details of techniques taken from the published works of many authors, and from his own visits to and correspondence with several laboratories. The text is divided into four parts. I, "Materials", describes electrophysiological apparatus and accessories; II, "Actographs", reports the methods used over the past 25 years to measure insect activity; III, "Free-Moving and Tethered Preparations", describes the procedures selected researchers have used to record from nerve and muscle in cockroaches, locusts, crickets and flies; IV, "Organ and Tissue Preparations", emphasizes heart, gut and salivary gland preparations, methods of recording from cockroach ventral nerve cord, and nerve-muscle preparations of locust and cockroach leg. There is a reference section, containing selected literature prior to 1977 , an appendix listing suppliers and an index. The book is the first in a series to be concerned with problems of experimental entomology.

Ideally, a book that emphasizes methodology should be accurate, clear, concise and well-organized, and be designed to make its contents readily accessible to the reader. Insect Neurophysiological Techniques falls far short of these ideals. The text is wordy, and almost awash with minutiae. Often it strays from the topic at hand, and large sections are misplaced or mistitled. There is a wealth of detail, but it is often repetitive or 